\title{
Should clinical trials be approached differently for rare cancers?
}

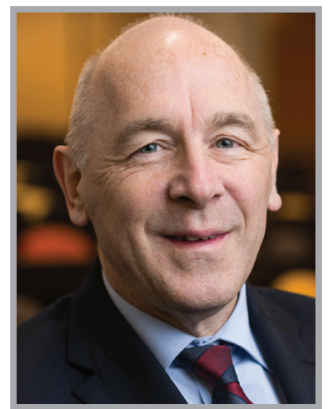

Ian Olver*

\author{
“...the methodologies to help compensate for the \\ small patient numbers associated with rare \\ cancers should be utilized to help provide \\ evidence to optimize treatments."
}

First draft submitted: 19 January 2016; Accepted for publication: 28 January 2016; Published online: 4 March 2016
One definition of rare cancers is that with an incidence of less than six cases per 100,000 population each year [1]. Identifying 198 cancers as rare leads to the estimate that they constitute $22 \%$ cancers [2]. In the USA, the National Cancer Institute uses a definition of $<15$ per 100,000 which translates to rare cancers accounting for $25 \%$ of cancer-related deaths. The survival of patients with rare cancers is poorer than that associated with common cancers but they show some of the highest response rates to molecularly targeted therapies because there is less interpatient variability in the pattern of genetic mutations than with more common cancers [3].

The traditional path for clinical trials for conventional chemotherapy has been to commence with a dose-finding Phase I trial which escalates doses in cohorts of patients until a maximum tolerated dose (MTD) is reached and the dose-limiting toxicities identified. A dose is then identified to test for efficacy in a Phase II trial in a group of patients with a particular cancer. If the new drug shows sufficient efficacy, it is compared with standard therapy in a Phase III randomized controlled trial. Here the patients are randomly allocated to avoid selection bias and distribute both known and unknown prognostic factors equally between the two treatment arms being studied, to help ensure that any differences found are due to the drugs being tested.

Randomized trials often require hundreds of patients to obtain sufficient power to identify clinically meaningful differences. The low incidence of rare cancers makes this approach problematic. This is part of the reason that less research is published on the treatment of these cancers. Different trial designs can address this problem, as can increasing accrual by forming partnerships such as the International Rare Cancers Initiative [4].

\section{Trial designs for rare cancers}

Even small Phase I trials in rare cancers can recruit less patients by using adaptive designs like a Bayesian continuous reassessment design. Here, the escalated dose to be given to the next patient is based on the cumulative toxicity data from all of
*Sansom Institute for Health Research, University of South Australia, P5-06e Playford Building, City East Campus, GPO Box 2471, Adelaide, SA 5001, Australia; Tel.: +61 883022 934; Fax: +61 883022 842; ian.olver@unisa.edu.au

\section{KEYWORDS}

- Bayesian • chemotherapy • PARP inhibitor $\bullet$ Phase I $\bullet$ Phase II

- Phase III • randomization $\bullet$ rare cancers $\bullet$ type I error $\bullet$ type II error
"The survival of patients with rare cancers is poorer than that associated with common cancers but they show some of the highest response rates to molecularly targeted therapies...”

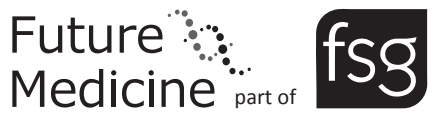


“A more speculative future approach to clinical trials in rare cancers or uncommon subtypes of common cancers, goes beyond just modifying clinical trial

design to circumvent the impracticality of

randomized Phase III trials in these patients." the patients recruited into the trial up until that time $[5,6]$.

There are also strategies which can be used in single-arm Phase II trials to reduce the size of patient populations needed to decide whether to continue testing a drug. For example, two stage designs using objective response $(50 \%$ or more tumor shrinkage) as an end point employ early stopping rules. One rule may test for futility after a small group (typically 14 patients) has been accrued and stop the accrual if the true tumor response rate is below a specified value and therefore the null hypothesis, that the drug is inactive, is accepted. Subsequently, designs also allow early stopping of a single-arm study at a specified level of efficacy [7].

The greatest challenge for trial design in rare cancers is where there are insufficient patient numbers to mount a randomized Phase III trial. One strategy is to perform a randomized Phase II trial to provide some prospective randomized evidence of the relative efficacy between new treatments, or between one or more new treatments and a standard treatment, to aid decisions about continued development. It may help select from two or more drugs in the randomized Phase II, based on their relative efficacy or if there is little difference in efficacy, on relative toxicities. The smaller randomized Phase II also allows a further judgment of whether accrual to a Phase III would be feasible [4].

Just performing the randomized Phase II without proceeding to a Phase III means accepting the chance of greater errors, particularly type I errors; that is incorrectly rejecting the null hypotheses by falsely concluding that a drug is effective when it is not. However in rare cancers, it may be pragmatic to accept the chance of both greater type I and the opposing type II error (failing to detect an effect that is present) because accrual will be low. This is a strategy already proposed in rare cancer trial designs [4].

As in the Phase I example, Bayesian designs for randomized Phase II studies allow the incorporation of external information, even subjective judgments of clinicians, to gauge the impact of a treatment. This incorporation of prior information allows a reduction in sample size. Unless Phase III trials are to follow, however, this results in a loss of certainty regarding the outcome. The strength of a randomized Phase II Bayesian design, however, compared with a single-arm study, is the ability to be able collect at least some information about the control treatment.
These designs also allow survival rather than response end points and are very helpful for rare cancers such as pancreatic cancer or uveal melanoma where there is no measure of objective response, or for investigation of cytostatic drugs where there are no meaningful objective responses expected [8].

Knowing the number of patients available for entry into a trial incorporating a Bayesian design allows estimation of the size of a treatment effect. The design also allows evaluation of the information that could be provided for several possible trial results and the chance of erroneous conclusions being reached $[4,8]$.

Where Phase III trials can be mounted in rare cancers, numbers can still be reduced. Conventional Phase III designs calculate the sample size before entering patients and are analyzed when the accrual is complete. They are powered to detect a difference that is thought to be clinically meaningful. It is possible to design a trial with a sequential design where interim analyses are performed and limits set, so the trial can be either terminated for futility or could continue if an upper limit for efficacy had not been met. This is just like with the single-arm studies and this method has been used, for example, in a trial of adjuvant therapy for the rare uveal melanomas [?].

\section{Further reasons to change clinical trial methodologies}

Having explored examples of designs which will allow for the reduced numbers of patients with rare cancers available to participate in trials, there are other factors which will drive a change in trial design for these patients. The first is the transition between histological classifications of tumors and genomic classifications. Trials are being conducted in tumors with the same genetic abnormalities irrespective of their histological subtype [3]. For example, PARP inhibitors which block DNA damage being repaired after chemotherapy were trialed in triple-negative breast cancers which carried $B R C A 1$ and $B R C A 2$ mutations. There are high-grade serous carcinomas of the ovary with genetic similarities to triple-negative breast cancer which also carry BRCA1 and BRCA2 mutations, therefore trials of PARP inhibitors have also commenced in that group of patients [10]. A further example is an ongoing trial of a BRAF inhibitor (vemurafenib) in patients with $B R A F$ V600 mutation-positive cancers that accepts patients with the mutation, irrespective of histological type [11]. 
Similarly, it is proposed that Phase I designs and end points will change for targeted therapies for cancers with rare genetic subtypes. It has been proposed that finding an maximum tolerated dose that has been appropriate for cytotoxic drugs may not be appropriate for immunotherapy where escalating doses may not be more effective. Here, not only should toxicity be assessed but an assessment of efficacy, perhaps by a biological response to a drug, may be desirable to identify a biologically optimal dose or even a minimum effective dose. Patients for these studies may require selection by whether their tumor displays the appropriate target of the therapy. Modeling of dose efficacy would proceed before the next cohort of patients was enrolled [12].

The other driver of new trial designs is that they will not just impact on rare cancers but on histologically common cancers which are being divided into increasingly smaller subgroups as they are grouped by their pattern of genetic mutations. It is these genetic changes that are providing the potential targets for the new targeted therapies and immunotherapies.

\section{Future clinical trial strategies}

A more speculative future approach to clinical trials in rare cancers or uncommon subtypes of common cancers, goes beyond just modifying clinical trial design to circumvent the impracticality of randomized Phase III trials in these patients. A Phase I trial would assess a biologically optimal dose in a patient population selected because it had the appropriate target for the treatment being trialed. Then a small Phase II trial of efficacy or even a series of case studies could affirm the proof of principle that the targeted therapy was effective. The therapy could then be released for clinical use in the targeted population. In health systems that digitize all their patient records, further refinements could be made, and the comparative effectiveness of the drug explored, by interrogating large international digital patient record databases which recorded the drug's use in practice [13].

Clinical trials are being approached differently as cytotoxic therapy gives way to targeted therapies and immunotherapies. Histologically rare cancers may share patterns of genetic mutations with more common cancers and this may help determine their eligibility for trials of molecularly targeted treatments. Even so, the methodologies to help compensate for the small patient numbers associated with rare cancers should be utilized to help provide evidence to optimize treatments.

\section{Financial \& competing interests disclosure}

The author has no relevant affiliations or financial involvement with any organization or entity with a financial interest in or financial conflict with the subject matter or materials discussed in the manuscript. This includes employment, consultancies, honoraria, stock ownership or options, expert testimony, grants or patents received or pending or royalties.

No writing assistance was utilized in the production of this manuscript.

\section{Reference}

1 Gratta G, van der Zwan JM, Casali PG et al. Rare cancers are not so rare: the rare cancer burden in Europe. Eur. J. Cancer 47, 2493-2511 (2011).

2 Panageas KS. Clinical trial design for rare cancers: why a less conventional route may be required. Expert Rev. Clin. Pharmacol. 8, 661-663 (2015).

3 Schott AF, Welch JJ, Verschraegen CF, Kurzrock R. The National Clinical Trials Network: conducting successful clinical trials of new therapies for rare cancers. Semin. Oncol. 42, 731-739 (2015).

4 Bogaerts J, Sydes MR, Keat N et al. Clinical trial designs for rare diseases: studies developed and discussed by the International Rare Cancer Initiative. Eur. J. Cancer 51, 271-281 (2015)
5 Sweeting M, Mander A, Sabin T. BCRM: Bayesian continual reassessment method designs for Phase I dose-finding trials. J. Stat. Softw. 54, 1-26 (2013).

6 Ji Y, Feng L, Liu P et al. Bayesian continual reassessment method for dose-finding trials infusing $\mathrm{T}$ cells with limited sample size. J. Biopharm. Stat. 22, 1206-1219 (2012).

7 Mander AP, Thomson SG. Two-stage designs under the alternative hypothesis for Phase II cancer clinical trials. Contemp. Clin. Trials 31, 572-578 (2010).

8 Cotterill A, Whitehead J. Bayesian methods for setting sample sizes and choosing allocation ratios in Phase II clinical trials with time-to-event endpoints. Stat. Med. 34, 1889-1903 (2015).

9 Whitehead J, Tishkovskaya S, O'Connor J, Domato B. Devising two-stage and multistage
Phase II studies on systemic adjuvant therapy for uveal melanoma. Invest. Ophthalmol. Vis. Sci. 53, 4986-4989 (2012).

10 Livraghi L, Garber JE. PARP inhibitors in the management breast cancer: current data and future prospects. BMC Med. 13, 188 (2015).

11 A study of Zelboraf (vemurafenib) in patients with BRAF V600 mutation-positive cancers. NCT01524978.

http://clinicaltrials.gov/show/NCT01524978

12 Mandekar S. Early phase trial designs and endpoints for targeted therapies in rare genotype subsets. Am. Soc. Clin. Oncol. Educ. Book 34, e107-e110 (2014).

13 Olver I. Reassessing rare cancers. Med.J. Aust. 203, 386 (2015). 